\title{
Scaling Behaviour of Chirality Dependent Domain Wall Pinning in Planar Nanowires
}

\author{
D.S. Eastwood*, L.K. Bogart and D. AtKinson \\ Department of Physics, Durham University, Durham, DH1 3LE, United Kingdom
}

\begin{abstract}
Micromagnetic modelling is used to establish the dimensional scaling dependence of the magnetic fields required for domain wall injection and chirality dependent pinning in planar permalloy nanowires with asymmetric structural notches. The wire width, thickness and notch constriction width are systematically varied whilst the axial magnetization is reversed under a globally applied magnetic field. A component of magnetic field transverse to the direction of propagation is applied throughout the reversal to control the domain wall chirality, and therefore determine the strength of pinning at the notch. We deduce that thicker wires with a narrower width cause larger depinning fields and stronger chirality dependent pinning, an observation relevant for domain wall memory applications, and particularly those exploiting chirality dependent pinning in nanowires.
\end{abstract}

PACS numbers: 75.60.Ch, 75.78.Cd, 81.07.Gf

\section{Introduction}

Magnetic nanowires can effectively act as conduits for propagating domain walls. They constitute the basis for several proposed magnetic memories based upon the controlled movement of magnetic domains [1-3]. The structure and dynamical deformation of domain walls propagating along a nanowire under the influence of a magnetic field [4] or a spin polarized current $[5,6]$ vary significantly with wire width and thickness and with wall velocity [7-9]. The micromagnetic structure of domain walls in nanowires can be classed as vortex or transverse, and assigned a chirality which describes the sense of rotation of the spins in the wall - clockwise or counterclockwise. It has been shown that the interaction of domain walls with structural features on one side of a wire which breaks the axial symmetry depends upon the chirality of the wall $[3,10]$, and this has been proposed as a mechanism for storing multiple bits of data in an elongated magnetic memory structure $[3,11]$.

In this paper we analyse micromagnetic simulations of the field induced formation, propagation and pinning of domain walls in notched nanowires of different widths, thicknesses and notch depths. We identify size constraints and write field margins which together allow strong discrimination between the write sequences needed to store different magnetization states in a magnetic memory based on chirality dependent pinning in nanowires.

In a linear quasi-infinite planar permalloy $\left(\mathrm{Ni}_{81} \mathrm{Fe}_{19}\right)$ nanowire magnetization is strongly influenced by the shape anisotropy, and the lowest energy magnetization

\footnotetext{
* corresponding author; e-mail: d.s.eastwood@durham.ac.uk
}

state occurs when there is single uniform magnetic domain directed along the wire axis and excluding any domain walls. A domain wall can be introduced either by attaching a low coercivity section to one end of the nanowire or by passing a current through an overlaid micron-scale strip line [12]. Domain walls formed in nanowires with high cross-sectional aspect ratios are confined by shape anisotropy to have local magnetization predominantly confined in the plane of the sample. In the limit of narrow, thin nanowires the most energetically favourable domain wall is a Néel-type transverse wall [8].

Here we use a $3 \mu \mathrm{m} \times 2 \mu \mathrm{m}$ permalloy rectangular "pad" at one end of the nanowire (as shown in Fig. 1), which switches magnetization under the application of a relatively low magnetic field. The domain wall is initially pinned at the pad-wire junction, and is injected into the wire when the axial field reaches a threshold $H_{\mathrm{inj}}$. If the domain wall chirality is incompatible with the notch geometry, it is strongly pinned at the notch until the axial field exceeds the depinning field $H_{\text {depin }}$.

There have been many studies on domain wall propagation in thin film nanowires, reporting several key findings. In small axial fields, domain wall motion is laminar and the velocity $v \propto 1 / \alpha$ where $\alpha$ is the damping coefficient $[8,13]$. In higher fields domain wall motion becomes turbulent and domain wall velocity can be significantly reduced. This is characterized as the Walker breakdown, in which precessional motion of local spins results in the formation of anti-vortex structures at wire edges, causing a periodic stalling and retrograde motion of the wall and acts to disrupt its chirality [14]. It is known that increasing $\alpha$ causes the threshold field for the onset of the Walker breakdown to increase [8], and that rougher edged wires can also inhibit the Walker breakdown by preventing the 


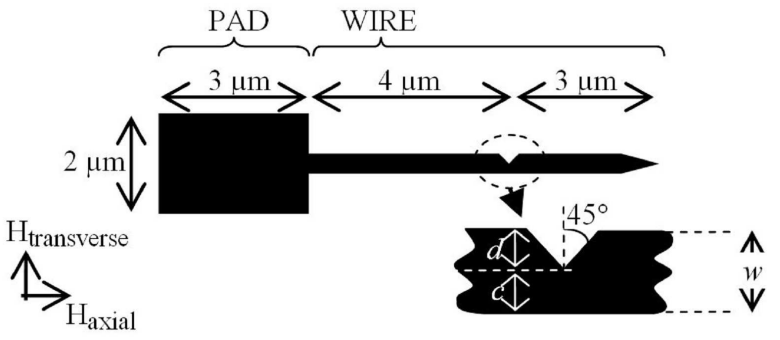

Fig. 1. Schematic of diagram of the planar nanowire and nucleation pad, showing the dimensions of the structure used in the micromagnetic simulations, and the orientation of the transverse and axial magnetic fields. The wire width, $w$, thickness, $t$, and notch depth, $d$, are systematically varied.

nucleation of anti-vortices and, therefore, increasing the domain wall velocity [15]. We chose to follow an alternative method described by Bryan et al. [16], inhibiting the Walker breakdown by applying a constant transverse field, $H_{\text {transverse }}= \pm 20$ Oe during motion. This creates a Zeeman interaction which encourages the domain wall to lie transverse to the wire axis with a central moment lying parallel to the transverse field. From previous experimental and theoretical work we know that different chiralities of wall are pinned at a notch on the upper edge of the wire with different strengths $[3,17]$. This transverse field also serves to set the initial chirality of the transverse wall during formation in the injection pad.

\section{Micromagnetic simulation methodology}

All of the micromagnetic simulations were performed using the public domain OOMMF software [18] which iteratively solves the Landau-Lifshitz equation for an assembly of discrete nanoscale "spins" under the influence of a spatially and temporally varying effective field. Here the simulations were quasi-static. The parameters used in the simulation were: damping parameter $\alpha=0.05$, saturation magnetization $M_{\mathrm{s}}=8 \times 10^{5} \mathrm{~A} \mathrm{~m}^{-1}$, exchange coefficient $A=13 \times 10^{-12} \mathrm{~J} \mathrm{~m}^{-1}$ and zero magnetocrystalline anisotropy, which is a reasonable representation of polycrystalline $\mathrm{Ni}_{81} \mathrm{Fe}_{19}$. In this work thin-film nanowires with thicknesses, $t$, between $3 \mathrm{~nm}$ and $20 \mathrm{~nm}$ were modelled using a $2 \mathrm{D}$ mesh of $3 \mathrm{D}$ spins. The cell size was $t \times 8 \mathrm{~nm} \times 8 \mathrm{~nm}$ which was slightly larger than the exchange length of 5-6 $\mathrm{nm}$, and produced results consistent with identical test simulations using smaller cells. The initial magnetic state was with the wire and pad saturated in the negative axial direction. In simulations the magnetic field was incremented in steps of +2.5 Oe until a domain wall was nucleated and had propagated along the full length of the wire. The simulation convergence criteria for each field step was $|\boldsymbol{M} \times \boldsymbol{H}| / M_{\mathrm{S}}^{2}<3 \times 10^{-5}$ i.e. when this condition was satisfied for every cell in the simulation it was assumed that the system was in equilibrium, and the axial field was then incremented further.

\section{Results and discussion}

Figure 2 shows, by way of example, the instantaneous magnetization states observed during the simulation of injection, propagation and pinning into a nanowire of width $200 \mathrm{~nm}$, thickness $10 \mathrm{~nm}$ and notch depth $100 \mathrm{~nm}$. Initially the wire is saturated in the negative direction by a large negative axial magnetic field. The axial field is then reversed in increments of 2.5 Oe in the presence of a constant transverse field of +20 Oe directed upwards in the figure. The chirality of the transverse domain wall which is formed at the pad-wire junction points upwards (Fig. 2a). At the injection field $H_{\text {inj }}=87.5$ Oe the transverse domain wall is injected from the pad into the wire and propagates along the wire at this field, retaining its transverse structure (Fig. 2b). Initially, the magnetization around the notch is constrained by local shape anisotropy and follows the edge of the nanowire, meaning that the spins to the right hand side of the notch are tilted downwards and oppose the leading spins in the domain wall. When the domain wall reaches the notch it is pinned just to the left of the constriction (Fig. 2c), as has been reported elsewhere $[10,19]$. As the axial field increases further, the domain wall remains pinned to the left of the notch, but simulations show that the exchange and magnetostatic energies increase causing the domain wall to deform and ultimately pass through the constriction. At $H_{\text {depinning }}=285$ Oe the energy supplied by the magnetic field is sufficient to overcome the potential barrier of the notch and the domain wall propagates along the wire before being annihilated at the sharp tip at the end of the wire. By contrast, when a domain wall is formed in the presence of a downwards pointing transverse field it has the opposite chirality, and the depinning field (from a notch of $50 \%$ wire depth) is less than the injection field, so the wall is not pinned and the wire is fully reversed at the injection field.

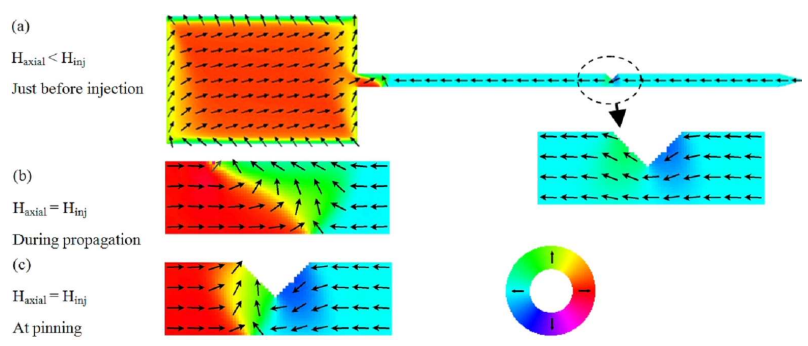

Fig. 2. A $200 \mathrm{~nm}$ wide, $10 \mathrm{~nm}$ thick nanowire with a $100 \mathrm{~nm}$ deep notch. (a) The magnetization state immediately prior to domain wall injection showing the local magnetization after the notch opposing the leading spins in the domain wall, (b) an "up" transverse wall in motion along the wire, and (c) the domain wall pinned at the notch.

For applications of nanowires in memory or sensor applications, the scaling of domain wall injection and depinning fields as a function of wire dimensions may be important. In the example given above the write field margins 
(the difference between the injection field of 87.5 Oe and the depinning field of 285 Oe) offers a large discrimination between pinning a domain wall at the notch or a fully reversing the wire. We will now consider the effect of changing the wire and notch dimensions.

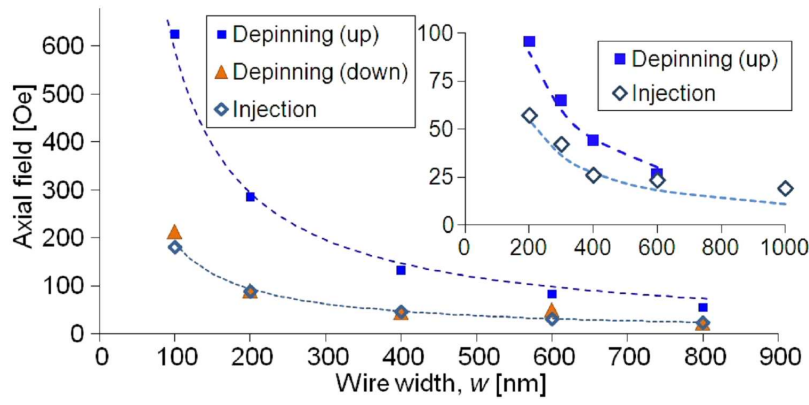

Fig. 3. Injection and depinning field as a function of wire width $(t=10 \mathrm{~nm}$, notch constriction $=50 \%)$ in the presence of a \pm 20 Oe transverse field. Dashed lines show $w^{-1}$ law fits to the injection and upwards depinning fields, demonstrating the inverse relationship between switching fields and wire width. Inset: for comparison, experimental values obtained by focussed magneto-optical Kerr effect (MOKE) measurements on $5 \mathrm{~nm}$ thick $\mathrm{Ni}_{81} \mathrm{Fe}_{19}$ nanowires of varying widths, showing the same $w^{-1}$ trend. Inset produced from raw data previously published in Ref. [20].

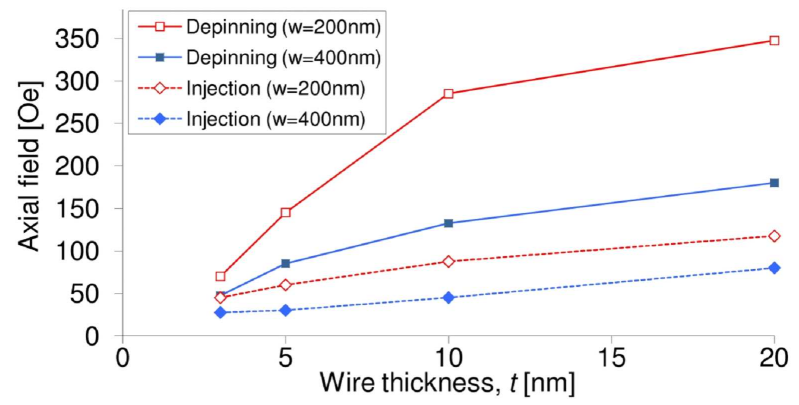

Fig. 4. Injection and depinning field as a function of wire thickness $(w=200$ or $400 \mathrm{~nm}$, notch constriction $=$ $50 \%$ ) in the presence of a +20 Oe transverse field. Lines are a guide to the eye only.

The injection and depinning fields for both chiralities of domain wall as a function of wire width are presented in Fig. 3 (all for a notch depth of $50 \%$ wire width). The most fundamental observation is that domain walls with a downwards moment formed by $H_{\text {transverse }}=-20 \mathrm{Oe}$ ("down walls") pass the notch at or close to the injection field, but that domain walls with an upwards moment formed in $H_{\text {transverse }}=+20$ Oe ("up walls") have a much higher depinning field, as has been demonstrated previously over a more limited range of wire widths [3]. Both injection and depinning fields decrease as the wire width increases, displaying an inverse relationship. This trend agrees with our earlier experimental measurements (on

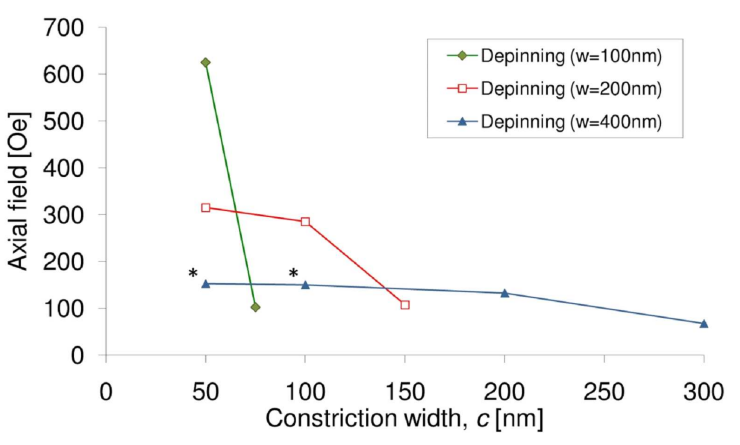

Fig. 5. Injection and depinning field as a function of notch depth for different wire widths $(t=10 \mathrm{~nm})$. Let us note that the points labelled "** indicate that domain walls are not observed to simply pass through the notch, but that a rotation of the wire segment after the notch occurs nucleating a new domain wall, followed by the ejection of a remaining $360^{\circ}$ domain wall. Lines are a guide to the eye only.

$5 \mathrm{~nm}$ thick wires) [20], which are replotted as an inset to Fig. 3. It can be understood by the decrease in magnetostatic energy (associated with stray field emanating from a transverse wall at the wire edges) and exchange energy (due to closely-packed misaligned spins) relative to the Zeeman interaction (the net scalar product of the local magnetic induction and magnetization). A further point of practical importance is the sharp increase of the depinning fields for wires only $100 \mathrm{~nm}$ wide. In memory devices reliant upon the generation of local magnetic fields by strip lines the current required to produce such high fields may cause significant device heating.

In the wider wires $(w \geq 600 \mathrm{~nm}, t=10 \mathrm{~nm})$ we observe the initial onset of the Walker breakdown when a vortex core enters the wire from the upper side. In the short wires we see that the magnetization moment of the leading edge of the vortex wall (the side which first interacts with the notch) is still determined by the transverse field direction, meaning that the local pinning interaction at the notch is similar. As the vortex wall is forced into and through the notch during depinning, the wall structure transforms to a transverse wall boundary.

The thickness dependence of injection and depinning fields (only shown for "up" walls) is shown in Fig. 4 for two widths of wire, $200 \mathrm{~nm}$ and $400 \mathrm{~nm}$. Thicker wires have larger injection and depinning fields for both widths of wire, but a simple power law is not observed. In thicker wires the tilting of magnetization out of the plane of the wire becomes less energetically expensive, and the domain wall structure no longer forms an ideal Néel wall. In very thick wires it may no longer be valid to exclude variation of magnetization through the wire thickness. One point of interest is that in a $3 \mathrm{~nm}$ thick wire (expected to contain an ideal Néel wall), a domain wall is injected into a $200 \mathrm{~nm}$ wire at approximately the same field as it is depinned from a $200 \mathrm{~nm}$ constriction created by a $50 \%$ notch in a $400 \mathrm{~nm}$ wire, indicating the dom- 
inant effect that the wire width has in controlling the wall depinning field in nanowires. In Fig. 5 the constriction width (i.e. the wire width minus the notch depth) is therefore used as the independent variable.

Figure 5 shows the influence of the notch constriction width on the strength of the pinning for three different widths of wire. As may be intuitively expected, wider constrictions offer a lower potential barrier to domain wall propagation for all widths of wire. The depinning fields for the two narrowest constrictions in the $400 \mathrm{~nm}$ wire (labelled "*") are low, because simulations show the nucleation of a second domain wall in the final segment of the wire, rather than simple passage of the domain wall through the constriction.

\section{Summary and conclusions}

Using micromagnetic simulations domain wall injection and chirality-dependent pinning and depinning in notched $\mathrm{Ni}_{81} \mathrm{Fe}_{19}$ nanowires have been surveyed as a function of wire thickness, width and notch depth. We observed strong chirality dependent pinning over a range of wire widths. As wire width, $w$, decreased from $800 \mathrm{~nm}$ to $100 \mathrm{~nm}$ the injection and depinning fields increased following a $1 / w$ trend. In contrast, as wire thickness decreased from $20 \mathrm{~nm}$ to $3 \mathrm{~nm}$ the injection and depinning fields also decreased. Shallower notches provided weaker pinning sites, but very deep notches in wide wires (400 nm) effectively prevented domain wall passage even under high axial fields, and a re-nucleation event occurred beyond the notch.

This study considered relatively short nanowires (because of the long computing time needed to model larger structures) and our results are directly applicable to magnetic elements $\approx 10 \mu \mathrm{m}$ in length, but the main conclusions on the pinning strength of notch constrictions are valid for much longer nanowire structures if the Walker breakdown is prevented. There are clear implications for a memory device based upon chirality dependent pinning in nanowires with multiple notches. The largest absolute margin between domain wall injection and the field at which chirality dependent pinning is overcome occurs in narrow, thick wires, but the generation of the large localized fields needed to saturate the narrowest wires is technically challenging. This may be compensated for by reducing the wire thickness, which will lower the injection and depinning fields.

\section{References}

[1] S.S.P. Parkin, M. Hayashi, L. Thomas, Science $\mathbf{3 2 0}$ 190 (2008).

[2] D.A. Allwood, G. Xiong, R.P. Cowburn, Appl. Phys. Lett. 89, 3 (2006).

[3] D. Atkinson, D.S. Eastwood, L.K. Bogart, Appl. Phys. Lett. 92, (2008).

[4] T. Ono, H. Miyajima, K. Shigeto, K. Mibu, N. Hosoito, T. Shinjo, Science 284, 468 (1999).

[5] L. Berger, J. Appl. Phys. 49, 2156 (1978).

[6] J.C. Slonczewski, J. Magn. Magn. Mater. 159, L1 (1996).

[7] D. Atkinson, D.A. Allwood, G. Xiong, M.D. Cooke, C.C. Faulkner, R.P. Cowburn, Nature Mater. 2, 85 (2003).

[8] Y. Nakatani, A. Thiaville, J. Miltat, J. Magn. Magn. Mater. 290-291, 750 (2005).

[9] A. Kunz, IEEE Trans. Magn. 42, 3219 (2006).

[10] L.K. Bogart, D. Atkinson, K. O'Shea, D. McGrouther, S. McVitie, Phys. Rev. B 79, 054414 (2009).

[11] D. Atkinson, D.S. Eastwood, Magnetic Structure with Multiple-Bit Storage Capabilities, Patent. WO2008090305 (2008).

[12] M. Hayashi, L. Thomas, C. Rettner, R. Moriya, X. Jiang, S.S.P. Parkin, Phys. Rev. Lett. 97, 207205 (2006).

[13] G.S.D. Beach, C. Nistor, C. Knutson, M. Tsoi, J.L. Erskine, Nature Mater. 4, 741 (2005).

[14] N.L. Schryer, L.R. Walker, J. Appl. Phys. 45, 5406 (1974).

[15] Y. Nakatani, A. Thiaville, J. Miltat, Nature Mater. 2, 521 (2003).

[16] M.T. Bryan, T. Schrefl, D. Atkinson, D.A. Allwood, J. Appl. Phys. 103, (2008).

[17] D. Petit, A.-V. Jausovec, D. Read, R.P. Cowburn, J. Appl. Phys. 103, 114307 (2008).

[18] M.J. Donahue, D.G. Porter, OOMMF User's Guide, Version 1.0, National Institute of Standards and Technology, Gaithersburg 1999.

[19] M. Klaui, H. Ehrke, U. Rudiger, T. Kasama, R.E. Dunin-Borkowski, D. Backes, L.J. Heyderman, C.A.F. Vaz, J.A.C. Bland, G. Faini, E. Cambril, W. Wernsdorfer, Appl. Phys. Lett. 87, 3 (2005).

[20] L.K. Bogart, D.S. Eastwood, D. Atkinson, J. Appl. Phys. 104, 033904 (2008). 